\title{
Natalizumab Associated PML in a Multiple Sclerosis Patient: Excellent Response to Minimal Intervention
}

Silvia R. Delgado ${ }^{1}$, Efrat Saraf Lavi ${ }^{2}$, Yesica A. Campos ${ }^{1}$, Melissa Ortega ${ }^{1}$, Kottil Rammohan ${ }^{1}$, William A. Sheremata ${ }^{1 *}$

${ }^{1}$ Departments of Neurology and Radiology, Miller School of Medicine University of Miami, CRB 13th Floor, Miami FL 33136, USA

${ }^{2}$ Departments of Neuroradiology, Miller School of Medicine University of Miami, CRB 13th Floor, Miami FL 33136, USA

"Corresponding author: William A. Sheremata MD, Emeritus Professor of Neurology, Miller School of Medicine University of Miami, CRB 13th Floor, Miami FL 33136, USA, Tel: (305) 243-2279; E-mail: wsherema@med.miami.edu

Received date: Jul 31, 2014, Accepted date: Sep 17, 2014, Publication date: Sep 22, 2014

Copyright: ( 2014 R. Delgado S, et al. This is an open-access article distributed under the terms of the Creative Commons Attribution License, which permits unrestricted use, distribution, and reproduction in any medium, provided the original author and source are credited.

\begin{abstract}
We present a case of a woman with natalizumab associated progressive multifocal leukoencephalopathy (PML) who developed an immune reconstitution inflammatory syndrome (IRIS) 9 weeks after natalizumab withdrawal. She responded well to a small dose of steroids intravenously, without plasma exchange, and has returned to her baseline status within one year of her initial symptoms and MRI abnormality.
\end{abstract}

Keywords: Multiple sclerosis; Natalizumab, PML, IRIS, Plasma exchange

\section{Introduction}

Management of natalizumab associated progressive multifocal leukoencephalopathy (PML) is challenging and there is no consensus based on prospective study [1,2]. The potential danger of inflammatory cerebral edema [3] accompanying the immune reconstitution inflammatory syndrome (IRIS) after drug withdrawal, and use of plasma exchange, prompted the use of high dose steroids $[4,5]$. However, steroids have been shown to inhibit the antiviral response [6], and plasma exchange (PLEX) results in an increase in the number of JCV copies [5].

We present a woman with natalizumab associated PML who developed an IRIS 9 weeks after natalizumab withdrawal. She responded well to a limited dose of steroids without oral taper or PLEX. The patient has returned to her baseline status within one year of her initial symptoms and MRI abnormality.

\section{Case Report}

A 62 year-old woman with a 27 -year history of multiple sclerosis (MS) treated with natalizumab for 5 years presented with transient word finding difficulty in April 2013 followed by resolution one month later; only to return and worsen in August 2013. Except for resolution of word finding difficulty, her neurological findings in May 2013 were unchanged. She had a known asymptomatic cavernous angioma of her right cerebellar hemisphere.

Upon her diagnosis 25 years previously she entered an IRB approved clinical study of human interferon-alpha (IFN-an) during which she experienced resolution of her neurological deficits and stabilized. After trial completion, and drug withdrawal, she relapsed and received a similar product, interferon- $\alpha$ n 3 (IFN- $\alpha$ n3; Alferon- $\mathrm{N}^{\star}$ ), off label prior to interferon- $\beta 1 b$; IFN- $\beta 1 b$ availability. She remained stable without disability until 2003 when she was diagnosed with a ductal carcinoma of her breast. Interferon was withdrawn prior to her chemotherapy. This consisted of doxorubicin (an anthracene compound) and cyclophosphamide. After completion she was placed on interferon- $\beta 1 \mathrm{a}$ (IFN- $\beta 1 \mathrm{a}) 44 \mu \mathrm{g} 3 \mathrm{X}$ weekly. She tolerated the drug poorly and experienced increased ataxia. Her cerebellar angioma remained unchanged. After a fall in 2008 that resulted in severe degenerative joint disease in her right knee she required a cane or a walker. She also experienced mild word-finding difficulty that did not progress. In November 2008, 5 years after her chemotherapy and subsequent IFN- $\beta 1$ a therapy she was switched to natalizumab.

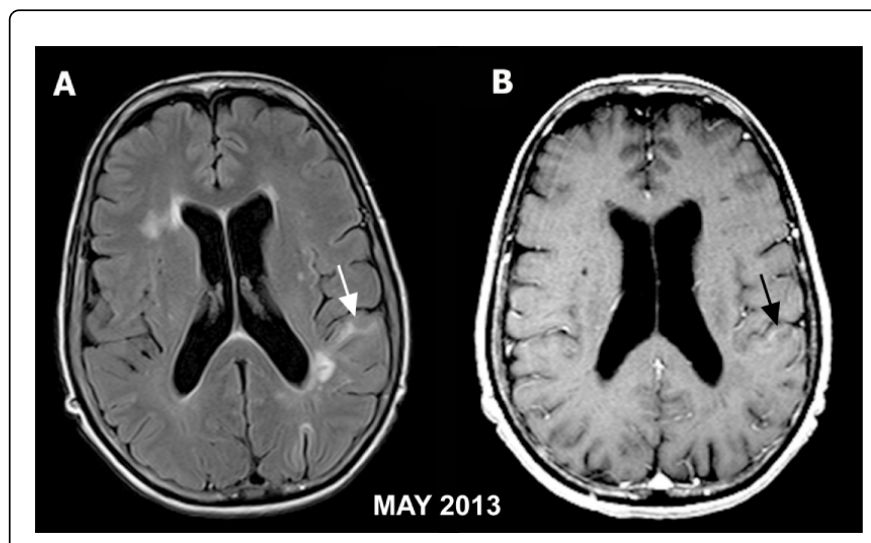

Figure 1: Magnetic resonance imaging evaluation of multiple sclerosis (MS) and progressive multifocal leukoencephalopathy (PML). After receiving 55 infusions of Natalizumab (May 2013). Axial FLAIR (A) and corresponding contrast enhanced T1W images (B) show MS lesions in the periventricular white matter and a new area of hyperintense FLAIR signal (white arrow) and faint enhancement (black arrow) in the left parietal subcortical white matter.

The patient remained stable on natalizumab until April 2013 when she complained of increased word-finding difficulty. Imaging studies in May 2013 were interpreted as unchanged. However, in retrospect the images showed the presence of a well-defined new lesion with slight gadolinium contrast (Gd) enhancement in the left parietal area (Figure 1). Re-examination in May 2013 revealed that her increased 
Citation: Delgado SR, Lavi ES, Campos YA, Ortega M, Rammohan K, et al. (2014) Natalizumab Associated PML in a Multiple Sclerosis Patient: Excellent Response to Minimal Intervention. J Mult Scler 1: 119. doi:10.4172/jmso.1000119

Page 2 of 4

word-finding difficulty had apparently resolved and she was otherwise unchanged. Importantly, the patient continued to perform in her professional capacity prior to and throughout her natalizumab treatment for MS. Her chart was annotated that she was JCV antibody "negative" in January 2013 and repeated testing was advised.

On presentation in the first week of September 2013, she stated that her speech deteriorated In August, a month prior. Her last dose of natalizumab had been received August 17th 2013. Her husband thought, in retrospect, the word finding difficulty began after her visit in May 2013. Blood drawn in the clinic, and reported one week later, revealed a high index of JC Virus antibody (3.8;). In the interval her speech deteriorated markedly and she was admitted to hospital.

Hospital Admission-10 September 2013, (23 days after her last dose of natalizumab); The patient exhibited marked word finding difficulty, anomia, right-left confusion, and an apraxia of her right arm. She had a mild right (central) facial paresis with a hemiparesis, greater in her right upper extremity. However, she was unable to walk even when unassisted.

Brain MRI on admission revealed increased T2 signal with "faint" Gd enhancement in the left temporal-parietal region (Figure 1 and Figure 2). In retrospect, in the May 2013 study (Figure 1) a small area of signal abnormality was identified corresponding to the central portion of the newly identified area of T2 signal abnormality in the left temporal-parietal region (Figure 2 and 3). Laboratory Studies (10SEP2013); Hematology and biochemical profiles were normal. Cerebrospinal fluid (CSF) contained 0 white and 233 red cells. The total protein was $35 \mathrm{mg} / \mathrm{dL}$ and IgG was $3.6 \mathrm{mg} / \mathrm{dL}$. The PCR for JC virus was spuriously recorded as "negative" while in the hospital. Appearing neurological stable, without evidence of JC virus in the CSF, and IRIS or any recognized contrast enhancing brain lesions, she was transferred to a rehabilitation facility.



Figure 2: Evolution of progressive multifocal leukoencephalopathy - Immune reconstitution inflammatory syndrome (PML-IRIS). Axial FLAIR and corresponding contrast enhanced T1W images through the temporal lobe. (A) Ten weeks after Natalizumab discontinuation and before steroid treatment (October 21, 2013), there is a new area of hyperintense FLAIR signal in the left temporal lobe (white arrows) with associated enhancement (black arrowheads). (B) Eleven weeks after Natalizumab discontinuation and after IV steroid therapy (October 29, 2013), there is decreased enhancement (black arrowheads) without change in FLAIR signal abnormality. (C) 6-month follow up after steroid treatment, there is further improvement with complete resolution of the enhancement.

Second hospitalization- 21October 2013; Clinically, the patient continued to have severe word finding difficulty and her findings resembling those she presented with one month earlier. New MRI studies revealed substantially increased abnormalities. (Figure $2 \mathrm{~A} \& \mathrm{~B}$ )
Summary of CSF findings; fluid obtained on 10 September 2014, on the first hospitalization was later reported to contain 97 JC virus copies/dL with a "cut-off" of 73. (Virucor). Confirmation of a significant number of JC Virus copies in the original specimen was 
Citation: $\quad$ Delgado SR, Lavi ES, Campos YA, Ortega M, Rammohan K, et al. (2014) Natalizumab Associated PML in a Multiple Sclerosis Patient: Excellent Response to Minimal Intervention. J Mult Scler 1: 119. doi:10.4172/jmso.1000119

Page 3 of 4

reported from NIH (17 copies with a "cut-off" of 11 copies). The CSF examination, on her second admission 32 days later, revealed 0 white and 26 red cells. Total protein was $39 \mathrm{mg} / \mathrm{dL}$ and the $\operatorname{IgG}$ index was 0.51 . No significant number of JC Virus copies was found in the CSF (37 with a "cut-off" of 40).

New Brain MRI revealed the presence of new areas of patchy Gd enhancement consistent with IRIS. These new areas of enhancement were seen in the left frontoparietal and temporal lobe, primarily involving the subcortical white matter. The signal changes were more confluent in the temporal lobe periventricular white matter. Corresponding increases of abnormal signal were evident in the FLAIR images. (Figure 2 and Figure 3).

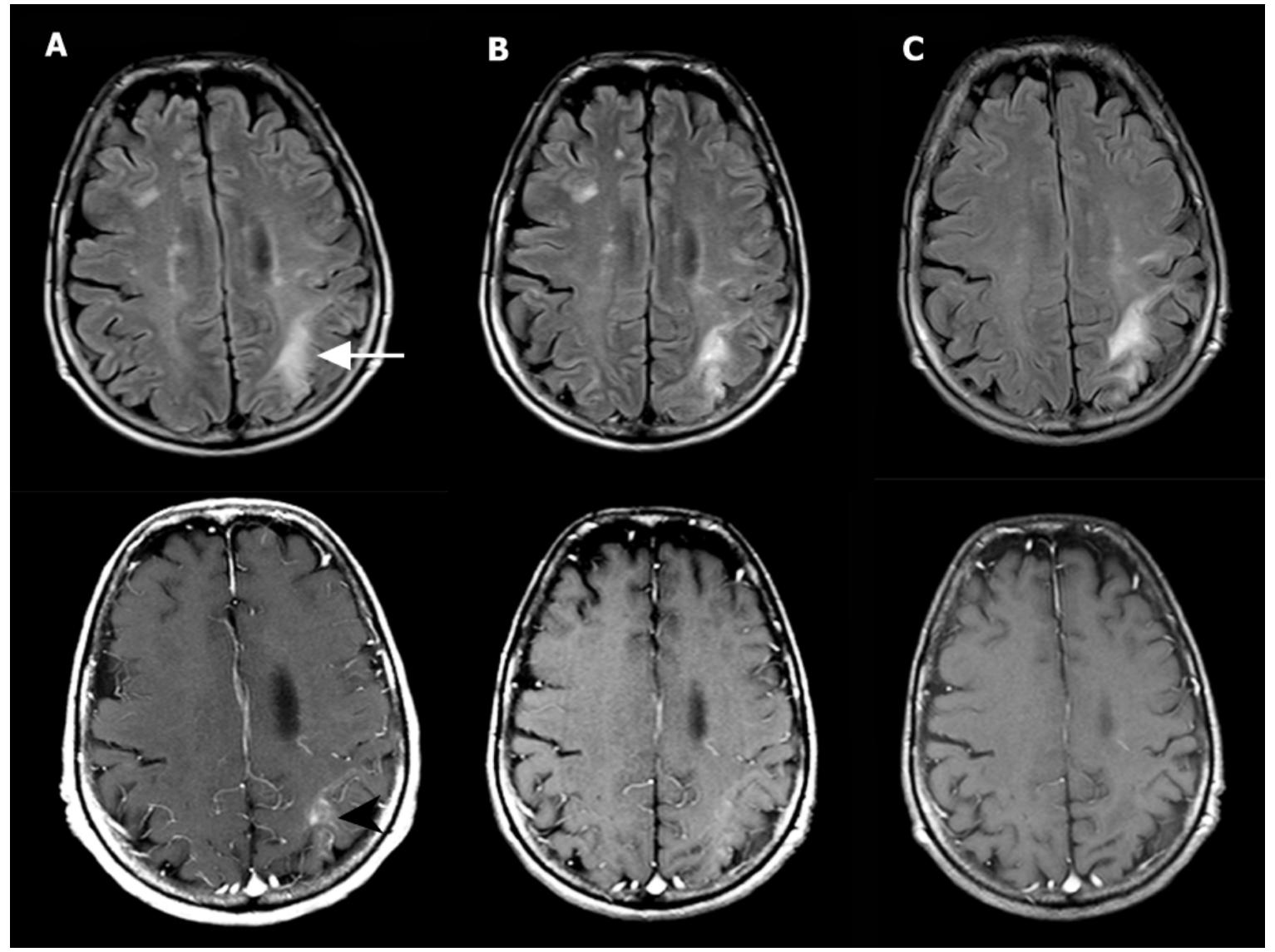

Figure 3: Evolution of progressive multifocal leukoencephalopathy - Immune reconstitution inflammatory syndrome (PML-IRIS). Axial FLAIR and corresponding contrast enhanced T1W images through the parietal lobe. (A) Ten weeks after Natalizumab discontinuation and before steroid treatment (October 21, 2013), there is further extension of the parietal lobe white matter lesion (white arrows) with associated enhancement (black arrowheads). (B) Eleven weeks after Natalizumab discontinuation and after IV steroid therapy (October 29, 2013), there is decreased enhancement without change in FLAIR signal abnormality. (C) 6-month follow up after steroid treatment, there is further improvement with complete resolution of the enhancement.

Hospital course; The patient received a total of $3 \mathrm{~g}$ methylprednisolone intravenously over 4 days with resolution of her hemiparesis and facial asymmetry. After 6 days, her speech difficulties improved remarkably she became able to converse without stopping and searching for words. She became able to use her right hand for tasks (such as dialing her cell-phone) and walk for a few steps without a cane.

11 Clinical Observations on Follow-up: The patient was reexamined on a monthly basis with additional brain MRI studies. Speech improved continuously with only slight word finding difficulty resembling her "pre-PML" status within 6 months of natalizumab withdrawal. Her ability to walk distances has improved but is limited primarily by her degenerative joint disease and her unwillingness to consider joint replacement. Progressive resolution of the PML/IRIS related Gd enhancing MRI lesions present at discharge was accomplished within 3 months of the appearance of IRIS.

Summary of the evolution of the clinical presentation of PML and brain MRI abnormalities (Figures 1-3) In May 2013, after 55 months of natalizumab therapy, a new hyperintense FLAIR signal abnormality, with Gd enhancement, was evident in the left parietal subcortical white matter (in retrospect). After 58 infusions of natalizumab, and 3 weeks after the last dose, with prominent clinical evidence of PML, extension of these areas of abnormality was seen. Nine weeks after natalizumab discontinuation (a total of 58 doses), and prior to steroid use, a new left temporal lobe lesion, further extension of the left parietal lobe lesion, with patchy enhancement of the new lesions was evident. 
Following steroid therapy serial imaging showed decreasing enhancement (not shown) with complete resolution of $\mathrm{Gd}$ enhancement at 6-months post steroids.

\section{Discussion}

Use of natalizumab implies an explicit risk of PML. The occurrence of this once fatal disorder does not have the implications initially associated with a diagnosis of PML. Earlier recognition and more effective management promise better outcomes for this once dreaded complication of immunosuppression. [2,5-10] We have described a woman with natalizumab-associated PML/IRIS who responded to minimal therapeutic intervention. This observation reawakens questions as to the best management of this serious disorder.

Occurrence of IRIS complicating PML prompted the use of steroids for management of accompanying cerebral inflammation and edema [1-5]. Following a 2010 conference in Germany, Hartung published a consensus statement recommending methyl-prednisolone, 1 or $2 \mathrm{~g}$ intravenously daily, for 5 days followed by a slow taper of oral steroids [3]. However, no prospective studies have been presented to establish their value $[7,8]$. Tan et al reviewed treatment outcomes of 40 patients with PML/IRIS managed with steroids and PLEX [5]. The value of steroids was unclear and it appeared that PLEX may adversely affect a subgroup. In the recent report by Fine et al, 17 patients developed PML after withdrawal of natalizumab but none died on follow-up regardless of their management [10]. Only 6 were confirmed to have had plasma exchange. Important evidence has been presented that steroids limit viral JC Virus clearance, [6] suggesting the need for a new approach to the management of IRIS [7-9].

Our patient did well despite having multi-lobar PML suggesting a poorer prognosis, although she had a relatively small number of JC Viral copies in her CSF. We question the value of using high dose steroids in the absence of life-threating brain edema.

\section{Acknowledgements}

The authors thank the staff of Jackson Memorial Hospital and the Miller School of Medicine for their assistance in patient care delivery and in retrieving data for this publication. We thank the scientific representatives from Biogen-Idec and Novartis in retrieving information relevant to PML/IRIS. No financial assistance was provided by any entity for this work.

\section{References}

1. Clifford DB, DeLuca A, Simpson DM, Arendt G, Giovannoni G et al. (2010) Natalizumab-associated progressive multifocal leukoencephalopathy in patients with multiple sclerosis. Lessons from 28 cases. Lancet Neurol 9:438-446.

2. Bloomgren G, Richman S, Hotermans C, Subramanyam M, Goelz S et al. (2012) Risk of natalizumab-associated progressive multifocal leukoencephalopathy N Engl J Med 368:1370-1380.

3. Metz I, Radue EW, Oterino A, Kümpfel T, Wiendl H et al. (2012) Pathology of immune reconstitution inflammatory syndrome in multiple sclerosis with natalizumab-associated progressive multifocal leukoencephalopathy. Acta Neuropathol; 123: 235-245.

4. Hartung HP, Berger J, Wiendl H (2011) Therapy for multiple sclerosis with monoclonal antibodies: updated recommendations for the use of natalizumab in the framework expert meetings. Akt Neurol 38: 2-11.

5. Tan IL, McArthur JC, Clifford DB, Major EO, Nath A (2011) Immune reconstitution inflammatory syndrome in natalizumab-associated PML. Neurology 77: 1061-1067.

6. Antoniol C, Jilek S, Schluep M, Mercier N, Canales M, et al. (2012) Impairment of JCV-specific T-cell response by corticotherapy: effect on PML-IRIS management? Neurology 79: 2258-2264.

7. Stefoski D, Ko M, Balabanov R (2012) Novel interventions with favorable resolution of natalizumab-induced progressive multifocal leukoencephalopathy (PML). Poster American Academy of Neurology Annual Meeting.

8. Ko M, Balabanov R, Stefoski D (2013) Facilitation of progressive multifocal leukoencephalopathy progression in natalizumab-treated multiple sclerosis patients by suppression of viral clearance with corticosteroids. Neurology 80: 1177.

9. Giacomini PS, Rozenberg A, Metz I, Araujo D, Arbour N, et al. (2014) Maraviroc and JC virus-associated immune reconstitution inflammatory syndrome. N Engl J Med 370: 486-488.

10. Fine AJ, Sorbello A, Kortepeter C, Scarazzini L (2014) Progressive multifocal leukoencephalopathy after natalizumab discontinuation. Ann Neurol 75: 108-115. 